\title{
Additional Effects of Nutritional Antioxidant Supplementation on Peripheral Muscle during Pulmonary Rehabilitation in COPD Patients: A Randomized Controlled Trial
}

\author{
Fares Gouzi (iD, ${ }^{1}$ Jonathan Maury, ${ }^{2,3}$ Nelly Héraud, ${ }^{3}$ Nicolas Molinari, ${ }^{4}$ Héléna Bertet, ${ }^{4}$ \\ Bronia Ayoub, ${ }^{1}$ Marine Blaquière, ${ }^{1}$ François Bughin, ${ }^{1}$ Philippe De Rigal, ${ }^{3}$ Magali Poulain, ${ }^{3}$ \\ Joël Pincemail, ${ }^{5}$ Jean-Paul Cristol (D, ${ }^{1}$ Dalila Laoudj-Chenivesse, ${ }^{2}$ Jacques Mercier, \\ Christian Préfaut, ${ }^{6}$ Pascal Pomiès $(\mathbb{D})^{2}$ and Maurice Hayot ${ }^{1}$ \\ ${ }^{1}$ PhyMedExp, Montpellier University, INSERM U1046, CNRS UMR 9214, Montpellier University Hospital, France \\ ${ }^{2}$ PhyMedExp, Montpellier University, INSERM U1046, CNRS UMR 9214, France \\ ${ }^{3}$ Pulmonary Rehabilitation Centers Les Cliniques du Souffle®, 5 Santé/Fontalvie Corporation, 66350 Toulouges, France \\ ${ }^{4} I M A G$, CNRS, Montpellier University, Montpellier University Hospital, Montpellier, France \\ ${ }^{5}$ Department of Cardiovascular Surgery and CREDEC, University of Liège, Sart Tilman University Hospital, 4000 Liège, Belgium \\ ${ }^{6}$ Montpellier University, France
}

Correspondence should be addressed to Fares Gouzi; f-gouzi@chu-montpellier.fr

Fares Gouzi and Jonathan Maury contributed equally to this work.

Received 2 December 2018; Accepted 24 February 2019; Published 17 April 2019

Academic Editor: Ioannis G. Fatouros

Copyright (c) 2019 Fares Gouzi et al. This is an open access article distributed under the Creative Commons Attribution License, which permits unrestricted use, distribution, and reproduction in any medium, provided the original work is properly cited.

\begin{abstract}
Background. Skeletal muscle dysfunction in patients with chronic obstructive pulmonary disease (COPD) is not fully reversed by exercise training. Antioxidants are critical for muscle homeostasis and adaptation to training. However, COPD patients experience antioxidant deficits that worsen after training and might impact their muscle response to training. Nutritional antioxidant supplementation in combination with pulmonary rehabilitation (PR) would further improve muscle function, oxidative stress, and PR outcomes in COPD patients. Methods. Sixty-four COPD patients admitted to inpatient PR were randomized to receive 28 days of oral antioxidant supplementation targeting the previously observed deficits (PR antioxidant group; $\alpha$-tocopherol: $30 \mathrm{mg} /$ day, ascorbate: $180 \mathrm{mg} /$ day, zinc gluconate: $15 \mathrm{mg} /$ day, selenomethionine: $50 \mu \mathrm{g} /$ day) or placebo (PR placebo group). PR consisted of 24 sessions of moderate-intensity exercise training. Changes in muscle endurance (primary outcome), oxidative stress, and PR outcomes were assessed. Results. Eighty-one percent of the patients $\left(\mathrm{FEV}_{1}=58.9 \pm 20.0\right.$ \%pred) showed at least one nutritional antioxidant deficit. Training improved muscle endurance in the PR placebo group $(+37.4 \pm 45.1 \%, p<0.001)$, without additional increase in the PR antioxidant group $(-6.6 \pm 11.3 \% ; p=0.56)$. Nevertheless, supplementation increased the $\alpha$-tocopherol $/ \gamma$-tocopherol ratio and selenium $(+58 \pm 20 \%, p<0.001$, and $+16 \pm 5 \%, p<0.01$, respectively), muscle strength $(+11 \pm 3 \%, p<0.001)$, and serum total proteins $(+7 \pm 2 \%, p<0.001)$, and it tended to increase the type I fiber proportion $(+32 \pm 17 \%, p=0.07)$. The prevalence of muscle weakness decreased in the PR antioxidant group only, from 30.0 to $10.7 \%(p<0.05)$. Conclusions. While the primary outcome was not significantly improved, COPD patients demonstrate significant improvements of secondary outcomes (muscle strength and other training-refractory outcomes), suggesting a potential "add-on" effect of the nutritional antioxidant supplementation (vitamins C and E, zinc, and selenium) during PR. This trial is registered with NCT01942889.
\end{abstract}




\section{Introduction}

Chronic obstructive pulmonary disease (COPD) is systematically associated with comorbidities [1]. Peripheral muscle dysfunction, characterized by reduced endurance, atrophy, and weakness, is a common comorbidity that negatively impacts prognosis [2]. Muscle atrophy and weakness are therefore targets of exercise training interventions in pulmonary rehabilitation (PR) [3]. However, the available evidence suggests that the muscle response to training is blunted in COPD patients, with either no or subphysiological responses regarding muscle strength, fiber size, and oxidative fibers $[4,5]$. Although still debated [2], oxidative stress and antioxidant deficits could impair the muscle response to training in COPD.

Oxidative stress is indeed a deleterious factor leading to muscle dysfunction and atrophy in COPD [2]. It results from an imbalance between excessive reactive oxygen species (ROS) production and a deficit in antioxidant defenses. In vitro experiments in COPD atrophic myotubes [6] match the clinical resting and exercise evidence [7]. Specifically, antioxidants play a key role as they improve muscle endurance [8] and atrophy in COPD [6].

Antioxidants also physiologically prevent muscle dysfunction and atrophy by buffering ROS [7], lowering systemic inflammation [9], and modulating cell functions [10]. Alphatocopherol, zinc, and selenium can protect the muscle against oxidative stress and inflammation in vitro and in vivo $[11,12]$ and are critical to muscle homeostasis [13]. The combination of antioxidant deficiencies impairs the muscle redox state [13] and has been implicated in aging sarcopenia [14] and inflammatory diseases $[15,16]$. Conversely, increasing the antioxidant content through dietary vitamin/trace element supplements [14-17] or regular moderate-intensity training [18] alone or in combination [19] has improved both systemic oxidative stress or inflammation and muscle function. Significantly, supplementation concomitant with training was efficient only in subjects with preexisting antioxidant deficits [19].

Last, in contrast to healthy individuals, COPD patients experience baseline antioxidant (vitamin $\mathrm{C}$, vitamin $\mathrm{C} / \mathrm{E}$ ratio, zinc, and selenium) deficits $[7,20]$, which worsen after training [5]. Thus, training alone did not reduce the oxidative damage and inflammation in patients [5]. Given the role of antioxidants in muscle dysfunction and the muscles' lack of improvement with training, antioxidants constitute a candidate for the blunted muscle response to training in COPD. Currently, only one study has tested the combination of training and antioxidant supplementation in COPD patients and showed no additional effect of the combination versus training alone. However, the single supplemented antioxidant did not increase its cellular target (i.e., the GSH/GSSG ratio) [21] or improve the other antioxidant deficits observed in COPD patients $[7,20]$.

In order to assess the potential of combining exercise training and antioxidant supplementation to target antioxidant deficits in stable COPD patients [20], we therefore conducted a randomized double-blind controlled trial during a PR program. We specifically tested the effects of oral antioxidant supplementation with vitamins and trace elements (i.e., vitamins $\mathrm{C}$ and E, zinc, and selenium) versus placebo on muscle endurance (primary outcome) and muscle strength, oxidative stress, inflammation, and PR outcomes (secondary outcomes).

\section{Material and Methods}

2.1. Study Design, Randomization, and Ethics. In this 28-day monocentric trial, COPD patients were randomly allocated (1:1 ratio) with permuted blocks of four patients, ensuring an equal number of patients receiving antioxidant supplementation (PR antioxidant group) and placebo (PR placebo group) during a PR program. The program included exercise training and followed the recommendations for chronic respiratory patients [3]. All patients received a detailed information letter about the study before providing written informed consent. Randomization was carried out after consent was obtained and centralized by computer software at the medical information department of Montpellier University Hospital. Investigators, PR caregivers, the statistician, and COPD patients were blinded to the nature of supplementation until trial completion. The study was conducted in accordance with good clinical practice and the Declaration of Helsinki. It was approved by the ethics committee Montpellier Sud-Mediterranée IV (no. 2011-A00842-39). The trial was preregistered in https://www.clinicaltrials.gov (ClinicalTrials.gov identifier: NCT01942889).

2.2. Participants. Stable COPD patients (40 to 78 years old) referred to the La Solane Pulmonary Rehabilitation Center (5 Santé /Fontalvie Corporation, F-66340, Osséja, France) were recruited. COPD was defined by the occurrence of dyspnea, chronic cough or sputum production, and/or a history of exposure to risk factors for the disease and postbronchodilatator $\mathrm{FEV}_{1} / \mathrm{FVC}<70 \%$, evaluated by plethysmography (Body Box 5500, Medisoft, Belgium) according to the validated methodology [1]. The exclusion criteria were as follows: exacerbations within the last month, unstable disease incompatible with a PR program, antioxidant supplementation (vitamins, trace elements, etc.) or use of drugs such as allopurinol and $\mathrm{N}$-acetylcysteine within the last month, and use of oral corticosteroids over the last six months. The BODE index was determined to assess global disease severity [7]. It was determined from the body mass index (B), the degree of airflow obstruction with $\mathrm{FEV}_{1}(\mathrm{O})$, dyspnea (D) as assessed by the Medical Research Council (MRC) scale, and exercise capacity (E) measured by the 6minute walk test (6MWT).

2.3. Interventions. In line with the current guidelines for pulmonary rehabilitation, 24 sessions of endurance exercise (stationary cycling, walking) over 28 days were proposed [3]. Endurance training intensity was individualized for each patient and corresponded to the target heart rate at the ventilatory threshold [4], as assessed during a maximal cardiopulmonary exercise test. For each patient, the ventilatory threshold was assessed blindly by two investigators. This intensity was continuously monitored with a heart rate monitor, and the workload was progressively increased. Each session lasted $1 \mathrm{~h}$ and $30 \mathrm{~min}: 45$ minutes of endurance training 
completed by 30 minutes of strength exercise (8-10 exercises, with sets of 10-15 repetitions) progressively increased using a perceived exertion scale (with a target of 5-6 on a 10-point scale) [4]. All sessions were supervised blindly by an experienced clinician. This exercise training was part of a multicomponent $\mathrm{PR}$ program. In order to promote long-term health behavior changes and long-term adherence, the PR also included dietary counseling, smoking cessation help, and educational interventions when needed [3].

Oral capsules of the antioxidant supplements or placebo were delivered to the patients daily by the nurses of the PR center. In accordance with the deficits observed previously in COPD patients [20], the antioxidant supplementation was associated with vitamin E ( $\alpha$-tocopherol: $30 \mathrm{mg} /$ day), vitamin C (ascorbate: $180 \mathrm{mg} /$ day), zinc gluconate (15 mg/day), and selenium (selenomethionine: $50 \mu \mathrm{g} /$ day). Although no vitamin E deficit was observed in the COPD patients, it was administered because of its synergistic effects with ascorbate [11]. Vitamin E was given as $\alpha$-tocopherol because this is the form preferentially absorbed by humans, and it has more antioxidant effects than other tocopherols or tocotrienols [11]. The nutritional supplementation doses were in line with (selenium) or above (vitamins $\mathrm{C}$ and $\mathrm{E}$ and zinc) the Recommended Dietary Allowances and Adequate Intakes (Food and Nutrition Board) [22].

\subsection{Outcomes}

2.4.1. Primary Outcome. The quadriceps endurance time (Qend, in seconds) may be more sensitive to interventions targeting the limb muscles, and the international guidelines therefore recommend its assessment in research studies [2]. The quadriceps endurance time was determined only for the dominant leg, as previously described by our group [8]. The patients performed knee extensions ( 6 movements per minute) with a workload to $30 \%$ of quadriceps maximal voluntary isometric contraction (QMVC) until exhaustion. Immediately after this test, they performed a QMVC to evaluate quadriceps fatigue, and a reduction in QMVC $>10 \%$ was necessary to validate the test, as previously published [8].

2.4.2. Secondary Outcomes. Secondary outcomes included changes in (1) quadriceps maximal voluntary isometric contraction (QMVC) and (2) oxidative stress (plasma vitamin and trace elements, lipid peroxidation, and GSH/GSSG ratio), as previously described [20]. QMVC was compared with the reference values. The predicted QMVC in $\mathrm{kg}$ was given by the following regression equation:

$$
\begin{aligned}
\text { QMVC }= & 56.2-(0.306 * \text { age in yrs })+(0.686 * \text { FFM in } \mathrm{kg}) \\
& -(0.156 * \text { height in } \mathrm{cm})-(3.42 \text { if female }) .
\end{aligned}
$$

The residual standard deviation from the analysis was $8.58 \mathrm{~kg}$, and patients with (observed-predicted QMVC)/ $8.58<-1.645$ were considered weak [23].

In addition, we assessed validated parameters of inflammation, muscle mass, exercise capacity, and nutritional status, as previously described [20]. Briefly, the muscle mass was assessed by bioelectrical multifrequency segmental impedance (Biacorpus RX Spectral; Medical HealthCare GmbH, Karlsruhe, Germany), as previously described [20]. Muscle biopsies were performed in the vastus lateralis of the quadriceps before and after the interventions as previously described [4]. Muscle fiber type and mean cross-sectional area (CSA) were assessed after immunohistochemistry on frozen sections, using anti-MHCI monoclonal antibodies [4]. The nutritional status was assessed by the evaluations of the body mass index (BMI) and body composition by bioelectrical multifrequency impedance. Total serum protide, albumin, and prealbumin were determined on venous blood collected in dry tubes. Samples were immediately centrifuged and frozen $\left(-80^{\circ} \mathrm{C}\right)$ until tested later. Total serum protide, albumin, and prealbumin were determined using an immune electrochemiluminescence assay on the Cobas 8000/e602 ${ }^{\circledR}$ immunochemistry system (Roche Diagnostics, Meylan, France). Last, the inflammation was assessed by a dosage of highly sensitive C-reactive protein (hsCRP) on venous blood collected in dry tubes. Samples were immediately centrifuged and frozen $\left(-80^{\circ} \mathrm{C}\right)$. Determination of CRP was run on the Cobas 8000/e502 ${ }^{\circledR}$ analyzer (Roche Diagnostics, Meylan, France) using the immunoturbidimetric method [24].

Treatment compliance was recorded daily by the nurses. Adverse events, the number of patients experiencing acute exacerbations of COPD (defined as an acute worsening of respiratory symptoms that results in additional therapy) [1], and additional medications (indication, cumulative systemic corticosteroid dose, and treatment length) were recorded and examined as covariables. Last, physical training (training session attendance, intensity) and micronutrient intake are influenced by pulmonary rehabilitation interventions, and therefore, these confounding variables were also recorded before and during the study.

2.5. Sample Size and Statistical Analysis. Sample size was calculated based on the change in Qend in seconds [8]. We estimated that with a sample of 28 patients per group evaluated for the primary outcome, the study had at least $80 \%$ power to determine the superiority of the PR antioxidant group compared with the PR placebo group. For the intention-to-treat analysis, the following assumption was made: Qend change of $180 \pm 120 \mathrm{~s}$ in the PR antioxidant group versus $90 \pm 120$ $\mathrm{s}$ in the PR placebo group. As we assumed a dropout rate of $10 \%$, we included 32 stable COPD patients per group. Quantitative data are presented as mean \pm standard deviation (SD), and qualitative data are described using proportions. To compare all the parameters between the PR placebo and PR antioxidant groups at baseline and to examine within-group relative variation, we used a Student $t$-test (or a Mann-Whitney for nonnormally distributed variables) for quantitative data and Pearson's chi-squared test for qualitative data. To assess the group effect on the relative variation, linear regressions were performed and covariable effects and their interactions were tested. If a confounder was identified, adjusted outcome analyses were performed by adding the confounder factor as a covariable of the test. For all analyses, the level of significance was set at $p<0.05$. Statistical analyses were performed on an intention-to-treat 
basis using $\mathrm{R}$ version 3.3.1 and SAS version 9.3 (SAS Institute, Cary, NC).

\section{Results}

3.1. Study Flow Chart and Population. Patients we enrolled between 3 June 2012 and 17 December 2014. As shown in the CONSORT flow diagram (Figure 1), 64 of the 389 screened COPD patients were randomized to receive placebo (PR placebo group, $n=32$ ) or antioxidant supplementation (PR antioxidant, $n=32$ ), 57 COPD patients completed the study and were included in the analysis, and 43 patients underwent muscle biopsy.

Participant characteristics and baseline measurements are shown in Tables 1 and 2 .

When measured values were compared with predicted values, the COPD patients $\left(49 \%\right.$ males, $\mathrm{FEV}_{1}=58.9 \pm 20.0$ $\%$ pred) were exercise-intolerant (symptom-limited oxygen uptake $\left(\mathrm{VO}_{2 \mathrm{sl}}\right)=60.3 \pm 16.4 \%$ pred and 6 -minute walking distance $(6 \mathrm{MWD})=80.0 \pm 15.1 \%$ pred $)$ and had muscle weakness (QMVC $=73.1 \pm 19.7 \%$ pred). Based on the reference values, $81 \%$ had at least one nutritional antioxidant deficit, with $26 \%, 24 \%$, and $67 \%$, respectively, deficient in vitamin $\mathrm{C}$, zinc, and selenium. As shown in Table 1, no significant differences were noted between the two groups for age, sex ratio, disease severity, body composition, or functional and skeletal muscle parameters. The GSH/GSSH ratio in the PR antioxidant group tended to be higher than that in the PR placebo group ( $454 \pm 281$ vs. $334 \pm 241$, respectively; $p=0.09$, Table 2). The characteristics of the COPD patients who underwent muscle biopsy did not differ significantly from those of the whole group (Table 3).

As shown in Table 4, PR did not differ between groups in terms of training, treatment compliance, or micronutrient intake. However, despite a similar number of acute exacerbations, the PR placebo patients received more short-term systemic corticosteroid treatment than the PR antioxidant patients $(31 \%$ vs. $6 \%$, respectively; $p<0.02)$.

3.2. Effect of the PR Program. The significant improvements in quadriceps endurance and exercise capacity (symptomlimited workload: $W_{\mathrm{sl}}$ and 6MWD) in the PR placebo patients show that the 4-week moderate-intensity training program was efficient. In addition, the PR placebo group showed improved outcomes that were driven not only by training (Tables 5 and 6), as we observed improvements in vitamin $\mathrm{C}(9.4 \pm 4.1$ to $12.8 \pm 3.3 ; p<0.001)$ and $\mathrm{GPx}$ $(45.4 \pm 10.7$ to $45.6 \pm 8.6 ; p<0.05)$.

\subsection{Outcome Analysis}

3.3.1. Primary Outcome Analysis. The primary outcome Qend showed no additional increase in the PR antioxidant group $(-6.6 \pm 11.3 \%$; $p=0.56)$. However, secondary outcome analysis revealed significant effects of the nutritional antioxidant supplementation (Tables 5 and 6).

3.3.2. Secondary Outcome Analyses. The corticosteroidadjusted increases in QMVC were significant (Figure 2) in the PR antioxidant group $\left(102 \pm 38\right.$ to $111 \pm 40 \mathrm{~N} \cdot \mathrm{m}^{-1}$; $p<0.001$ ), whereas this change was not significant in the PR placebo group $(114 \pm 51$ to $114 \pm 51 ; p=0.89)$. This resulted in a significant between-group difference in favor of antioxidant supplementation for QMVC $(p<0.001)$, after adjustment on the baseline difference and on the interaction between group and baseline values. The prevalence of muscle weakness decreased significantly (from 30.0 to $10.7 \%$; $p<0.05)$ in the PR antioxidant group but not significantly in the PR placebo group ( 41.7 to $37.5 \% ; p=0.56$ ), and this resulted in a significant between-group difference $(p<0.05)$.

We observed increases in the $\alpha$-tocopherol $/ \gamma$-tocopherol ratio and the selenium level in the PR antioxidant group that were significantly greater than those in the PR placebo group $(+51.1 \pm 10.7 \%, \quad p<0.001$, and $+16.2 \pm 4.5 \%, \quad p<0.01$, respectively). We found significant and positive correlations between the change in QMVC and the change in the $\alpha$-tocopherol $/ \gamma$-tocopherol ratio $(r=0.39 ; p<0.05)$ and between the change in QMVC and the change in selenium $(r=0.40$; $p<0.05$ ) (Figure 3). However, the change in markers of oxidative damage, such as lipid peroxidation, was not significant in the PR antioxidant group, and the magnitude of change did not differ from that in the PR placebo group $(1.1 \pm 13.1 \%$, $p=0.93)$. There was a significant difference in the corticosteroid-adjusted change in the GSH/GSSG ratio. When the baseline values were taken into account, however, the change in the PR antioxidant group did not differ from that in the PR placebo group $(-82 \pm 61 \% ; p=0.19)$.

Although antioxidant supplementation did not significantly improve the muscle mass or fiber cross-sectional area, the proportion of the type I muscle fiber increased in the PR antioxidant group $(34.4 \pm 13.6 \%$ to $38.9 \pm 16.0 \%$; $p<0.05)$, whereas this change was not significant in the PR placebo group $(41.4 \pm 11.9 \%$ to $42.1 \pm 11.6 \%$; $p=0.89)$. This resulted in a trend for type I fiber proportion $(p=0.07)$, depicted in Figure 2.

Last, Tables 5 and 6 show the effect of antioxidant supplementation on serum total proteins versus placebo $(+6.5 \pm$ $2.4 \% ; p<0.01)$ but no significant effect of supplementation on other nutritional statuses or exercise capacity parameters.

\section{Discussion}

In this randomized double-blind, placebo-controlled trial, nutritional antioxidant supplementation (vitamins $\mathrm{C}$ and $\mathrm{E}$, zinc, and selenium) failed to further improve the patients' quadriceps endurance (primary outcome). However, this study demonstrates additional improvements of three secondary outcomes (antioxidant deficits, QMVC, and serum total protein) and a trend toward increased type I fiber proportion with supplementation versus placebo during PR.

With the exception of the GSH/GSSG ratio, the two groups were comparable at baseline, particularly for the muscle function parameters. Specifically, the factors involved in COPD muscle dysfunction, like physical activity level, tobacco smoking, and vitamin/trace element intake [2], did not significantly differ between the randomized groups. Moreover, blinding was maintained throughout the study, which ensured comparability. Indeed, as shown in Table 4, the duration and compliance with treatment; the number, 


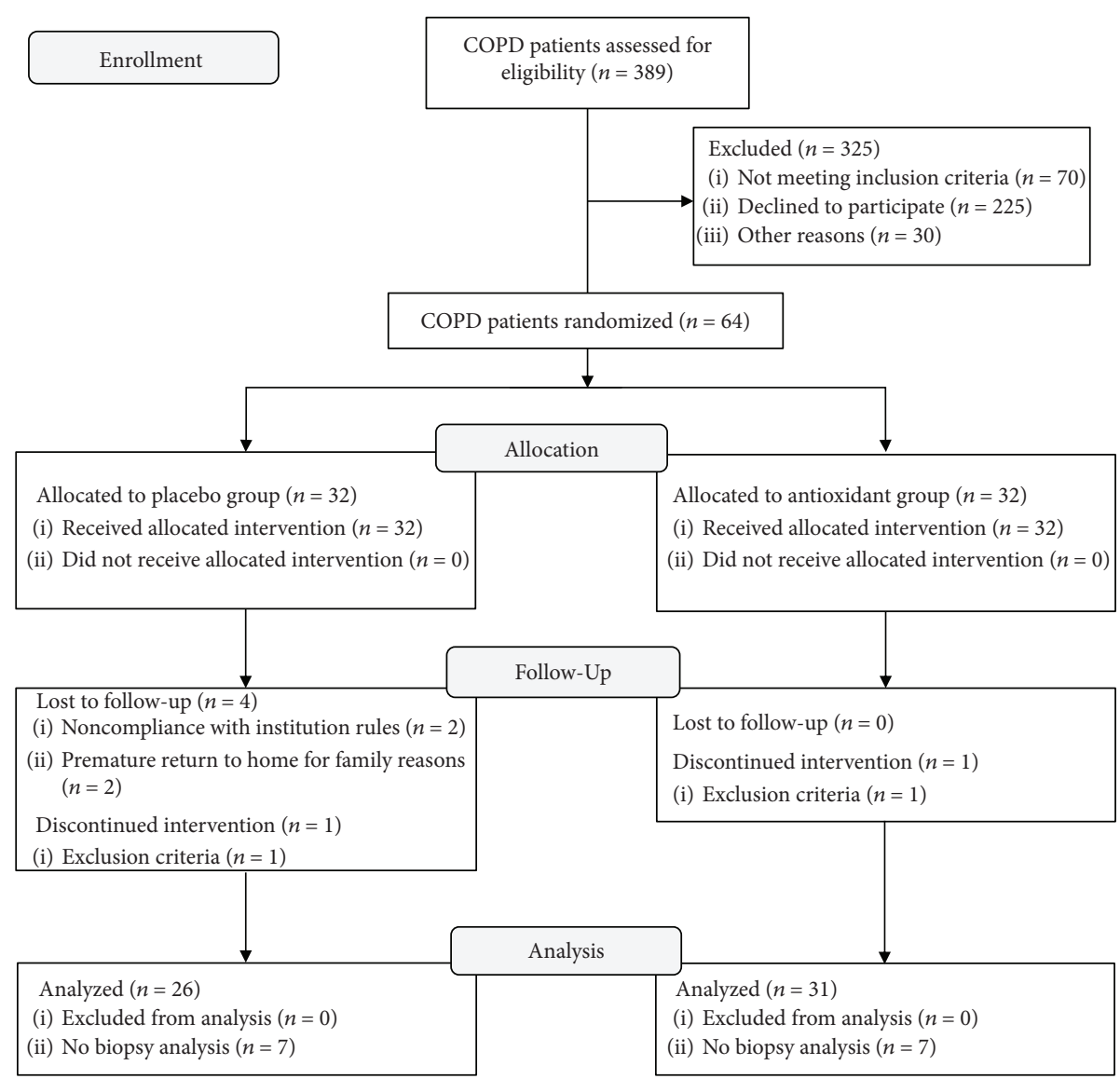

FIgURE 1: CONSORT diagram of the COPD patient recruitment.

type, and intensity of the training sessions; and the micronutrient intake did not differ between groups. The tendency toward more patient dropouts in the placebo group $(p=0.052)$ was not related to the disease or the intervention (instead, to noncompliance with institutional rules and family reasons). Adverse events and acute exacerbations did not differ between groups. The only confounding factor was a higher prevalence of oral corticosteroid prescriptions in the placebo group $(30.8 \%$ vs. $6.45 \%$; $p<0.05)$. Given the positive correlation between short-term corticosteroid treatment and muscle strength [25], and its impact on oxidative stress and inflammatory parameters, a post hoc adjustment was performed for all secondary outcome muscle measures.

4.1. Impact of the Antioxidant Supplementation on the Serum Antioxidants. At baseline, more than $60 \%$ of the COPD patients had dietary intakes of vitamin $\mathrm{C}$, vitamin $\mathrm{E}$, and zinc below the recommendations, with the only exception being selenium. Moreover, we observed a baseline deficit in nutritional antioxidants in these patients, reproducing our previous findings [20]. After training, we found no increases in most of the enzymatic and nonenzymatic antioxidants in the $\mathrm{PR}$ placebo group, in agreement with previous studies in COPD [5]. However, vitamin C and GPx were increased in this group, consistent with the dietary counseling and education delivered during $\mathrm{PR}$, as indicated by the high vitamin $\mathrm{C}$ and selenium intake in this nonsupplemented group
( $161 \pm 71 \%$ and $171 \pm 57 \%$ of the recommended dietary allowance, respectively). These increases in vitamin $\mathrm{C}$ and GPx may in part explain the lack of additional vitamin $C$ improvement in the PR antioxidant group. Yet this latter group showed significant additional improvement in the $\alpha$ tocopherol $/ \gamma$-tocopherol ratio and plasma selenium, indicating that supplementation was efficient [26]. Zinc was also improved in the supplemented group, although the tendency for additional improvement versus placebo $(p=0.096)$ was not significant after adjustment on corticosteroid use $(p=0.390)$, which increases the plasma zinc level [24] and may thus have masked the effect of zinc supplementation in our study.

4.2. Impact of Antioxidant Supplementation on Muscle Function during $P R$. Exercise training was efficient because the increase in $6 \mathrm{MWD}(+44 \pm 38 \mathrm{~m} ; p<0.001)$ was clinically significant in $62 \%(16 / 26)$ of the PR placebo patients [27]. Qend improved in both groups, and the adjusted difference between groups was not statistically significant $(p=0.56)$. Although we opted for the most sensitive muscle parameter to study pharmacological [28] and nonpharmacological interventions [4] targeting limb muscles [2], the sample size was based on the observed improvement in Qend after short-term antioxidant supplementation alone [8], which was the closest data available at the time for the design of our combined antioxidant and training study. However, 
TABLE 1: Baseline clinical, functional, and muscle characteristics of the randomized study population.

\begin{tabular}{|c|c|c|c|}
\hline & PR placebo group $(n=26)$ & PR antioxidant group $(n=31)$ & $p$ value \\
\hline Age (years) & $61.1 \pm 8.7$ & $62.4 \pm 6.5$ & 0.55 \\
\hline Sex ratio $(\mathrm{W} / \mathrm{M})$ & $13 / 13$ & $16 / 15$ & 0.90 \\
\hline BMI $\left(\mathrm{kg} / \mathrm{m}^{2}\right)$ & $25.3 \pm 4.7$ & $25.0 \pm 4.2$ & 0.78 \\
\hline Fat-free mass index $\left(\mathrm{kg} / \mathrm{m}^{2}\right)$ & $17.3 \pm 2.4$ & $17.4 \pm 2.3$ & 0.85 \\
\hline Muscle mass index $\left(\mathrm{kg} / \mathrm{m}^{2}\right)$ & $7.9 \pm 1.6$ & $8.0 \pm 1.5$ & 0.74 \\
\hline $\mathrm{FEV}_{1}(\% \text { pred })^{*}$ & $62 \pm 27$ & $57 \pm 17$ & 0.41 \\
\hline $\mathrm{FEV}_{1} / \mathrm{FVC}$ ratio* & $43 \pm 14$ & $41 \pm 10$ & 0.99 \\
\hline BODE score & $1.9 \pm 1.7$ & $2.2 \pm 1.9$ & 0.60 \\
\hline Breathlessness (MRC score) & $1.2 \pm 0.9$ & $1.3 \pm 1.2$ & 0.81 \\
\hline Tobacco consumption (pack years) & $40 \pm 18$ & $45 \pm 26$ & 0.63 \\
\hline Physical activity level score & $6.0 \pm 6.7$ & $5.7 \pm 4.9$ & 0.85 \\
\hline 6MWD (m) & $508 \pm 106$ & $504 \pm 112$ & 0.96 \\
\hline 6MWD (\%pred) & $79 \pm 17$ & $81 \pm 16$ & 0.64 \\
\hline$W_{\text {sl }}($ watt $)$ & $70 \pm 30$ & $60 \pm 25$ & 0.17 \\
\hline$W_{\mathrm{sl}}(\%$ pred $)$ & $52 \pm 19$ & $51 \pm 18$ & 0.84 \\
\hline $\mathrm{VO}_{2 \mathrm{sl}}(\mathrm{ml} / \mathrm{min} / \mathrm{kg})$ & $16.6 \pm 4.0(n=18)$ & $14.1 \pm 3.4(n=7)$ & 0.16 \\
\hline $\mathrm{VO}_{2 \mathrm{sl}}(\%$ pred $)$ & $66 \pm 16(n=18)$ & $56 \pm 15(n=7)$ & 0.16 \\
\hline $\mathrm{VO}_{2}$ at $\mathrm{VT}(\mathrm{ml} / \mathrm{min} / \mathrm{kg})$ & $10.1 \pm 2.7$ & $9.5 \pm 2.77$ & 0.42 \\
\hline QMVC (N·m) & $114 \pm 51$ & $114 \pm 51$ & 0.52 \\
\hline QMVC (\%pred) & $72.8 \pm 21.9$ & $74.0 \pm 21.6$ & 0.39 \\
\hline Qend (s) & $355 \pm 158$ & $343 \pm 117$ & 0.75 \\
\hline Type I muscle fiber \% & $41 \pm 12$ & $34 \pm 14$ & 0.09 \\
\hline Type I muscle fiber CSA $\left(\mu \mathrm{m}^{2}\right)$ & $5497 \pm 1689$ & $4810 \pm 1824$ & 0.22 \\
\hline Non-type I muscle fiber CSA $\left(\mu \mathrm{m}^{2}\right)$ & $4307 \pm 1740$ & $4118 \pm 1408$ & 0.84 \\
\hline All muscle fiber CSA $\left(\mu \mathrm{m}^{2}\right)$ & $4839 \pm 1589$ & $4369 \pm 1549$ & 0.34 \\
\hline
\end{tabular}

${ }^{*}$ Postbronchodilatation. Results are expressed as mean \pm SD. Definition of abbreviations: COPD: chronic obstructive pulmonary disease; PR: pulmonary rehabilitation; W/M: women/men; BMI: body mass index $\left(\mathrm{kg} / \mathrm{m}^{2}\right) ; \mathrm{FEV}_{1}$ (\%pred): forced expiratory volume in 1 second; BODE index: body mass index, airway obstruction, dyspnea, exercise capacity index (6MWD); MRC: Medical Research Council; 6MWD (m): six-minute walking distance; $W_{\mathrm{sl}}$ : symptomlimited power output; $\mathrm{VO}_{2 \mathrm{sl}}$ : symptom-limited oxygen uptake; VT: ventilatory threshold; QMVC $\left(\mathrm{N} \cdot \mathrm{m}^{-1}\right)$ : quadriceps maximal voluntary contraction expressed in Newtons; Qend (s): quadriceps endurance time expressed in seconds; CSA: cross-sectional area expressed in $\mu \mathrm{m}^{2}$.

given the large magnitude of Qend improvements in both the $\mathrm{PR}$ placebo and PR antioxidant groups $(+37 \pm 45 \%$ and $+24 \pm 43 \%$, respectively), a ceiling effect of the traininginduced increase in muscle function may have been reached, as recently reported for "add-on therapies" to training [29]. This effect was perhaps all the more notable as the PR placebo group was clearly not a training-only group but instead benefited from other interventions (such as dietary counseling and education). This ceiling effect was not observed for other secondary outcomes (except for the 6MWD), as shown by the nonsignificant changes observed in the PR placebo group. Conversely, the additional $11 \%$ improvement in QMVC in the PR antioxidant group was statistically significant and significantly correlated with changes in the $\alpha$-tocopherol $/ \gamma$-tocopherol ratio and selenium. This result is in agreement with previous studies showing that the greatest QMVC benefits have been observed in those with the largest antioxidant correction [19]. This further benefit of the "addon" antioxidant supplementation is a striking result, as the improvements appear inconsistent in other PR studies on resistance training (alone or combined with endurance training) [30] and neuromuscular electrostimulation [31], and on balance, it caused no significant harm. Moreover, the QMVC improvement may be clinically significant because the proportion of COPD patients with muscle weakness decreased significantly from 30.0 to $10.7 \%$ in the PR antioxidant group, which was from a population of COPD patients attending a "real-life" PR program and showing a prevalence of muscle weakness similar to that of published cohorts [23]. Given the prognostic value of muscle weakness [2], we speculate that a similar decrease in its prevalence would be clinically relevant in large-scale studies with long-term outcome assessment. Last, given the heterogeneity of the antioxidant deficits in our COPD patients, a personalized antioxidant supplementation tailored to the precise antioxidant status of each individual [32] could also constitute a relevant perspective for further muscle improvement during training in COPD patients. 
TABLE 2: Baseline serum oxidative stress and inflammation characteristics of the randomized study population.

\begin{tabular}{|c|c|c|c|}
\hline & PR placebo group $(n=26)$ & PR antioxidant group $(n=31)$ & $p$ value \\
\hline Vitamin C ( $\mu \mathrm{g} / \mathrm{ml})(\mathrm{W}: 8.6-18.8 ; \mathrm{M}: 6.2-15.2)$ & $9.5 \pm 4.1$ & $10.8 \pm 4.7$ & 0.29 \\
\hline$\alpha$-Tocopherol (mg/l) (8.0-15.0) & $14.1 \pm 3.0$ & $15.7 \pm 4.5$ & 0.12 \\
\hline$\gamma$-Tocopherol (mg/l) & $1.03 \pm 0.33$ & $1.18 \pm 0.55$ & 0.75 \\
\hline$\alpha$-Tocopherol $/ \gamma$-tocopherol ratio & $14.6 \pm 4.0$ & $15.2 \pm 6.0$ & 0.99 \\
\hline Vitamin $\mathrm{C} / \alpha$-tocopherol $(0.59-1.19)$ & $0.71 \pm 0.34$ & $0.71 \pm 0.33$ & 0.97 \\
\hline Selenium $(\mu \mathrm{g} / \mathrm{ml})(94-130)$ & $84.2 \pm 14.0$ & $87.6 \pm 14.6$ & 0.37 \\
\hline Copper (mg/ml) (W: 0.8-1.55; M: 0.7-1.40) & $1.13 \pm 0.26$ & $1.11 \pm 0.21$ & 0.76 \\
\hline Zinc (mg/ml) $(0.70-1.20)$ & $0.82 \pm 0.12$ & $0.79 \pm 0.13$ & 0.30 \\
\hline Copper/zinc ratio (1.14-1.29) & $1.41 \pm 0.42$ & $1.45 \pm 0.37$ & 0.45 \\
\hline GSH/GSSG ratio (111-747) & $334 \pm 241$ & $454 \pm 281$ & 0.09 \\
\hline GPx (UI/gHb) (30-55) & $45.4 \pm 10.7$ & $49.3 \pm 11.7$ & 0.20 \\
\hline SOD (UI/gHb) (785-1570) & $1308 \pm 351$ & $1286 \pm 250$ & 0.84 \\
\hline Lipid peroxidation $(\mu \mathrm{mol} / \mathrm{l})(<432)$ & $652 \pm 283$ & $625 \pm 309$ & 0.74 \\
\hline LDLox (UI/l) (28-70) & $59 \pm 21$ & $61 \pm 18$ & 0.44 \\
\hline hsCRP (mg/l) & $5.5 \pm 6.3$ & $6.8 \pm 10.9$ & 0.57 \\
\hline Total serum protides (g/l) & $68.8 \pm 5.8$ & $66.6 \pm 5.5$ & 0.13 \\
\hline Serum albumin $(\mathrm{g} / \mathrm{l})$ & $47.4 \pm 4.7$ & $46.2 \pm 8.3$ & 0.84 \\
\hline Serum prealbumin $(g / l)$ & $0.278 \pm 0.069$ & $0.293 \pm 0.069$ & 0.47 \\
\hline
\end{tabular}

Results are expressed as mean \pm SD. In the first column, we specify the lower and upper limits of reference values obtained in a large healthy subject cohort, as previously described [10]. Definition of abbreviations: PR: pulmonary rehabilitation; M: male; W: women; GSH: reduced glutathione; GSSG: oxidized glutathione; SOD: superoxide dismutase; GPx: peroxidase glutathione; LDL: oxidized low-density lipoprotein.

TABLE 3: Characteristics of the COPD patients who underwent muscle biopsy.

\begin{tabular}{|c|c|c|c|c|c|c|}
\hline & $\begin{array}{l}\text { PR placebo group } \\
(n=26)\end{array}$ & $\begin{array}{l}\text { Biopsy PR placebo group } \\
\qquad(n=19)\end{array}$ & $p$ value & $\begin{array}{l}\text { PR antioxidant group } \\
(n=31)\end{array}$ & $\begin{array}{c}\text { Biopsy PR antioxidant } \\
\text { group }(n=24)\end{array}$ & $p$ value \\
\hline Age (years) & $61 \pm 9$ & & & $62 \pm 6$ & & \\
\hline Sex ratio $(\mathrm{W} / \mathrm{M})$ & $13 / 14$ & $11 / 8$ & 1.00 & $17 / 15$ & $13 / 11$ & 1.00 \\
\hline BMI & $25.4 \pm 4.7$ & $24.6 \pm 4.3$ & 0.54 & $24.8 \pm 4.3$ & $25.8 \pm 4.1$ & 0.39 \\
\hline $\mathrm{FEV}_{1}$ (\%pred) & $62 \pm 27$ & $61 \pm 32$ & 0.67 & $55 \pm 18$ & $51 \pm 18$ & 0.91 \\
\hline 6MWD (\%pred) & $78 \pm 17$ & $78.1 \pm 17$ & 0.95 & $81 \pm 14$ & $84 \pm 13$ & 0.58 \\
\hline $\mathrm{VO}_{2 \mathrm{sl}}(\%$ pred $)$ & $64 \pm 16$ & $64 \pm 17$ & 0.90 & $56 \pm 16$ & $55 \pm 16$ & 0.84 \\
\hline QMVC (\%pred) & $90 \pm 24$ & $99 \pm 21$ & 0.18 & $87 \pm 14$ & $87 \pm 15$ & 0.97 \\
\hline$\alpha / \gamma$-Tocopherol & $15.7 \pm 4.1$ & $14.6 \pm 4.0$ & 0.39 & $15.2 \pm 6.0$ & $15.9 \pm 6.1$ & 0.69 \\
\hline Selenium & $85.4 \pm 14.2$ & $84.9 \pm 14.2$ & 0.91 & $88.3 \pm 14.3$ & $89.8 \pm 13.2$ & 0.70 \\
\hline Qend (s) & $357 \pm 155$ & $367 \pm 174$ & 0.82 & $345 \pm 116$ & $359 \pm 122$ & 0.66 \\
\hline Delta QMVC (\%) & $+3 \pm 11 \%$ & $+1 \pm 11 \%$ & 0.60 & $+9 \pm 9 \%$ & $+10 \pm 9 \%$ & 0.79 \\
\hline $\begin{array}{l}\text { Delta } \alpha / \gamma- \\
\text { tocopherol }(\%)\end{array}$ & $-4 \pm 24 \%$ & $-9 \pm 24 \%$ & 0.49 & $+53 \pm 47 \%$ & $+54 \pm 51 \%$ & 0.93 \\
\hline Delta selenium (\%) & $+2 \pm 17 \%$ & $0 \pm 17 \%$ & 0.61 & $+20 \pm 14 \%$ & $+20 \pm 14 \%$ & 0.92 \\
\hline
\end{tabular}

Results are expressed in mean \pm SD. Definition of abbreviations: W/M: women $/ \mathrm{men}$; BMI: body mass index $\left(\mathrm{kg} / \mathrm{m}^{2}\right)$; FEV 1 (\%pred): forced expiratory volume in 1 second; $6 \mathrm{MWD}(\mathrm{m})$ : six-minute walking distance; $\mathrm{VO}_{2 \mathrm{sl}}$ : symptom-limited oxygen uptake; QMVC $\left(\mathrm{N} \cdot \mathrm{m}^{-1}\right)$ : quadriceps maximal voluntary contraction expressed in Newtons; Qend (s): quadriceps endurance time expressed in seconds; CSA: cross-sectional area expressed in $\mu \mathrm{m}^{2}$.

4.3. Muscle Pathways Induced by the Nutritional Supplementation during PR. Evidence of the greater benefit of nutritional intervention plus exercise training as opposed to combined training and placebo is scarce $[2,29]$, and our study is the first to show the greater improvement in muscle function with dietary antioxidants versus placebo in combination with exercise training. For example, the addition of targeted nutrition combining vitamin D/omega3 or 
TABLE 4: Confounding factors during the study of the randomized study population.

\begin{tabular}{|c|c|c|c|}
\hline & PR placebo group $(n=26)$ & PR antioxidant group $(n=31)$ & $p$ value \\
\hline Systemic corticosteroid treatment & $8 / 18(31 \%)$ & $2 / 29(6 \%)$ & 0.02 \\
\hline Duration of corticosteroid treatment (days) & $4 \pm 1.4$ & $5 \pm 1.41$ & 0.33 \\
\hline \multicolumn{4}{|l|}{ Antioxidant/placebo supplementation } \\
\hline Duration (days) & $26.4 \pm 1.8$ & $26.7 \pm 0.7$ & 0.59 \\
\hline Capsule consumption $(n)$ & $112 \pm 3$ & $112 \pm 2$ & 0.91 \\
\hline Compliance (\%) & $99 \pm 2$ & $99 \pm 1$ & 0.37 \\
\hline \multicolumn{4}{|l|}{ Exercise training intensity } \\
\hline $\mathrm{VO}_{2 \mathrm{sl}}$ at VT/pred. $\mathrm{VO}_{2 \mathrm{sl}}(\%)$ & $42 \pm 12$ & $42 \pm 16$ & 0.44 \\
\hline \multicolumn{4}{|l|}{ Number of exercise training sessions } \\
\hline (i) stationary cycling & $8 \pm 2$ & $8 \pm 2$ & 0.83 \\
\hline (ii) walking & $8 \pm 3$ & $8 \pm 3$ & 0.39 \\
\hline (iii) strength building & $10 \pm 3$ & $10 \pm 4$ & 0.70 \\
\hline (iv) adapted physical activity & $10 \pm 4$ & $8 \pm 3$ & 0.11 \\
\hline \multicolumn{4}{|l|}{ Daily micronutrient intake } \\
\hline Vitamin C (mg/d) & $179 \pm 80$ & $201 \pm 81$ & \multirow{2}{*}{0.46} \\
\hline Vitamin C (\%RDA) & $161 \pm 71 \%$ & $183 \pm 74 \%$ & \\
\hline Vitamin E (mg/d) & $5.7 \pm 1.6$ & $5.3 \pm 1.7$ & \multirow{2}{*}{0.56} \\
\hline Vitamin E (\%RDA) & $47 \pm 13 \%$ & $44 \pm 14 \%$ & \\
\hline Zinc $(\mathrm{mg} / \mathrm{d})$ & $10.3 \pm 3.1$ & $10.0 \pm 2.8$ & \multirow{2}{*}{0.79} \\
\hline Zinc (\%RDA) & $97 \pm 32 \%$ & $87 \pm 24 \%$ & \\
\hline Copper $(\mathrm{mg} / \mathrm{d})$ & $1.4 \pm 0.5$ & $1.5 \pm 0.5$ & \multirow{2}{*}{0.60} \\
\hline Copper (\%RDA) & $86 \pm 29 \%$ & $88 \pm 31 \%$ & \\
\hline Selenium $(\mu \mathrm{g} / \mathrm{d})$ & $100.6 \pm 33.8$ & $98.3 \pm 29.8$ & \multirow{2}{*}{0.85} \\
\hline Selenium (\%RDA) & $171 \pm 57 \%$ & $162 \pm 49 \%$ & \\
\hline \multicolumn{4}{|l|}{ Exacerbations during interventions } \\
\hline Duration (days) & $5 \pm 3$ & $4 \pm 3$ & 0.49 \\
\hline
\end{tabular}

Results are expressed as mean \pm SD. Definition of abbreviations: PR: pulmonary rehabilitation; $\mathrm{VO}_{2 \mathrm{sl}}$ : symptom-limited oxygen uptake; VT: ventilatory threshold; RDA: Recommended Dietary Allowances and Adequate Intakes.

leucine/vitamin D/omega3 with $\mathrm{PR}$ showed no additional benefit for exercise capacity, muscle mass, or muscle function [33]. Supplementation with antioxidant whey protein combined with exercise training showed no effect on the muscle [21]. In our study, the increases in $\alpha$-tocopherol $/ \gamma$-tocopherol and selenium with supplementation increased total serum protein, a hallmark of the protein catabolic state in COPD [34]. Given the similar protein intake in the two groups, this may have been a consequence of reduced protein catabolism and inflammation [35]. Although not documented by hsCRP, lipid peroxidation, and the GSH/GSSG ratio, the involvement of reduced inflammation or oxidative stress in the additional improvement in muscle strength cannot be ruled out, because not all the plasma and muscle markers of inflammation and oxidative stress were tested.

However, vitamin $\mathrm{E}$ and selenium do not only act as ROS scavengers and/or anti-inflammatory molecules [11, 12], but also modulate several pathways and cell functions like proliferation and apoptosis (PI3K/Akt/PPAR $\gamma$ ) [11,
36]. In this context, the additional increase in type I fiber proportion in the supplemented group merits discussion. Although the adjusted difference only tended toward significance compared with the PR placebo group $(p=0.07$, power of the test: $37 \%$ ), the difference might have been statistically significant had the full randomized groups been taken into account. Indeed, given that the lack of change in type I fiber proportion in our training-only group reproduced our past data [4] and the findings of other studies [5], completing the biopsy analyses in the PR placebo group would likely have confirmed the effect of supplementation on type I fiber proportion. The extensive literature on muscle diseases caused by deficiencies in vitamin $\mathrm{E}$ and selenium intake [37] is in line with this effect of $\alpha$-tocopherol and plasma selenium on the muscle fiber type: white muscle disease, which is characterized by selective type I fiber destruction, is reversible with $\alpha$-tocopherol and selenium supplementation in animals [38] and humans [39, 40]. Selenium supplementation during training induces muscle mitochondrial biogenesis in animals and humans [41]. Given the baseline 


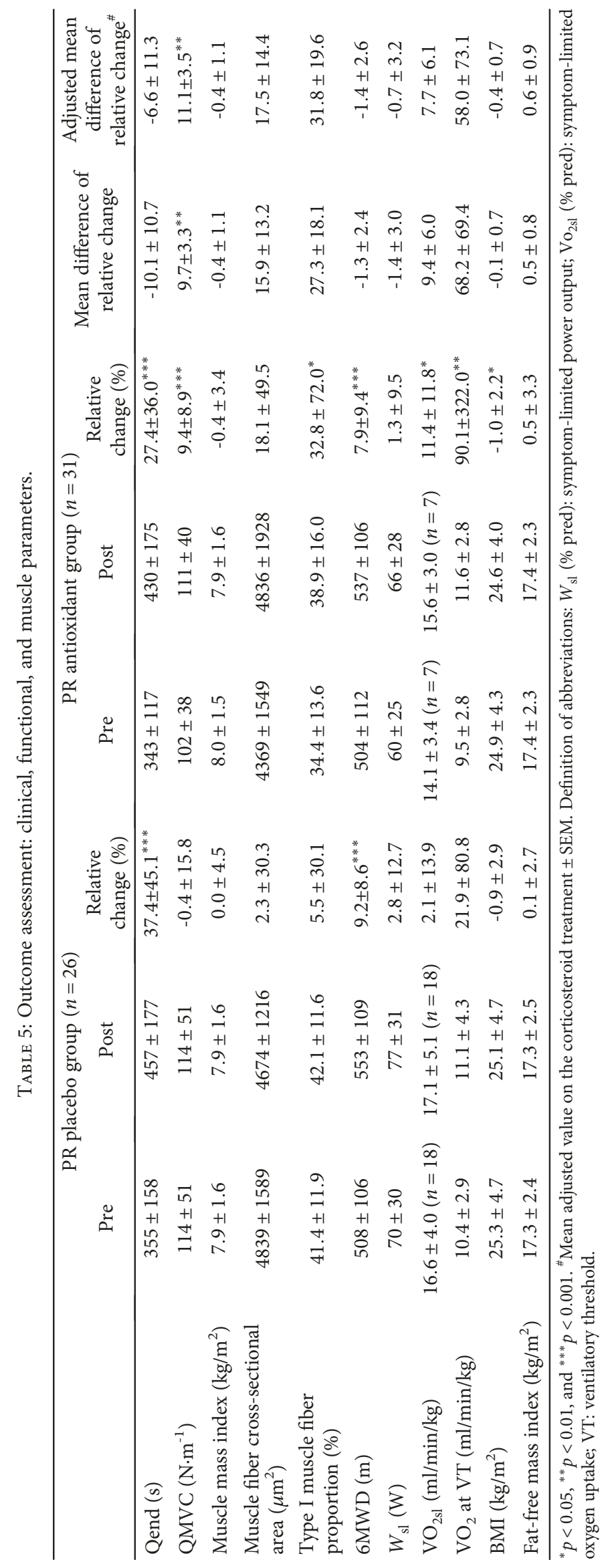




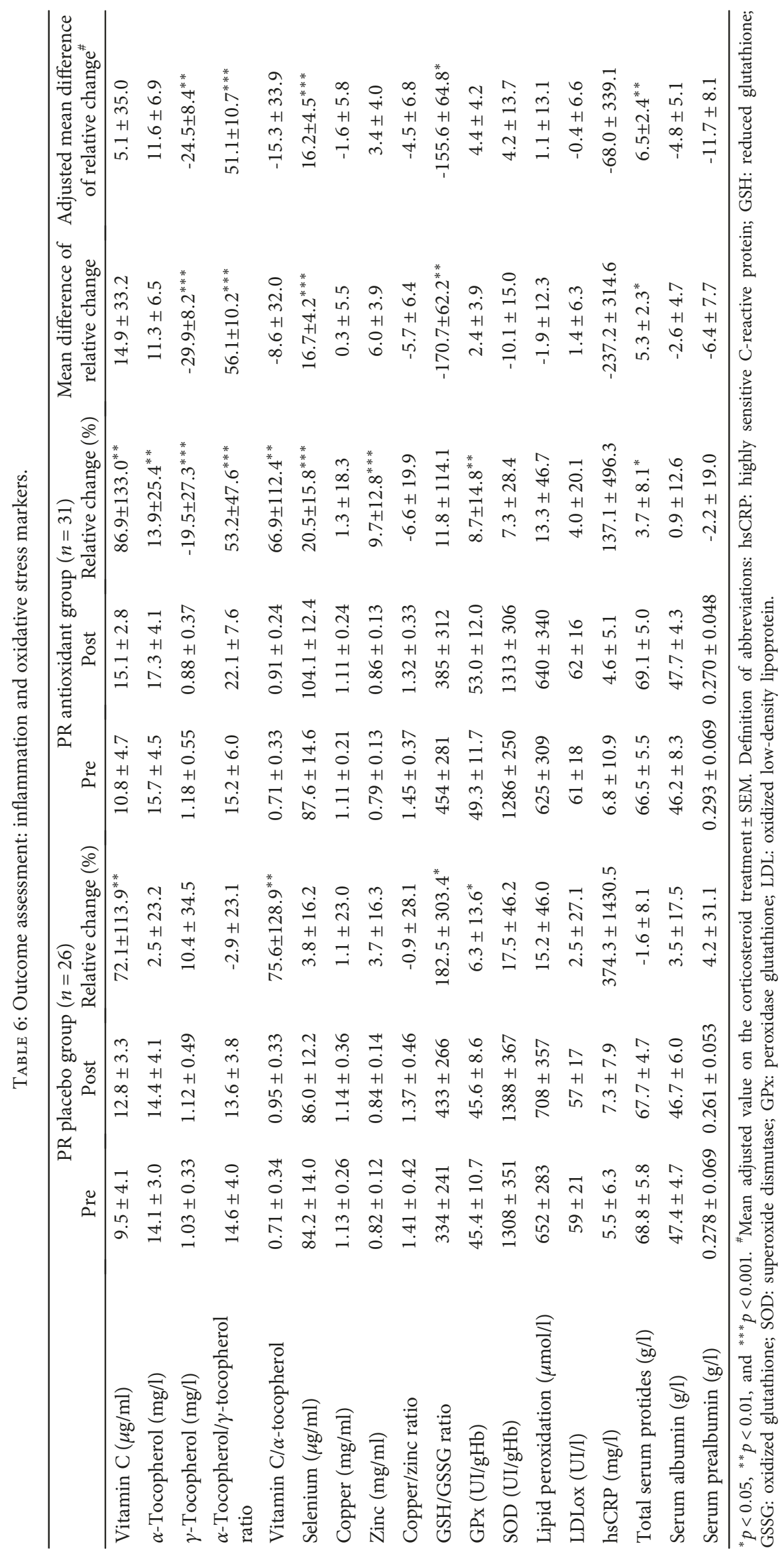




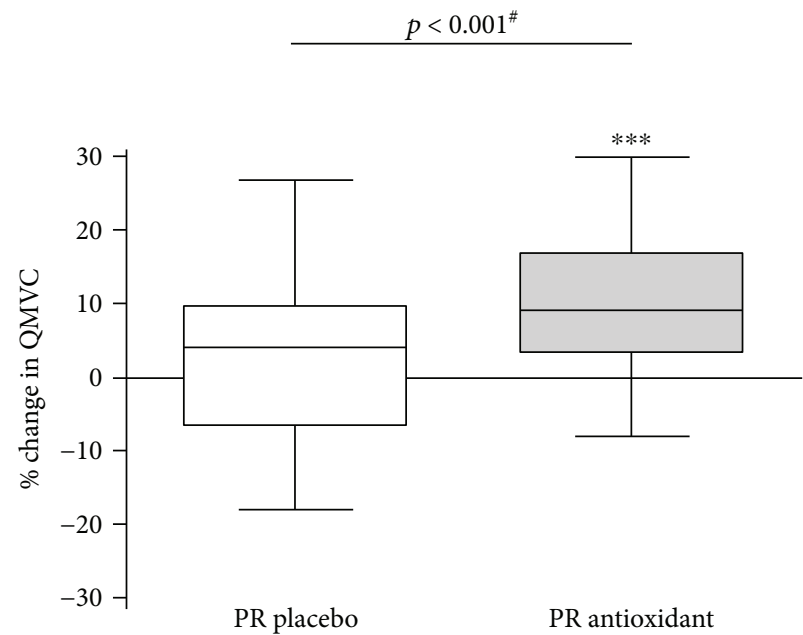

(a)

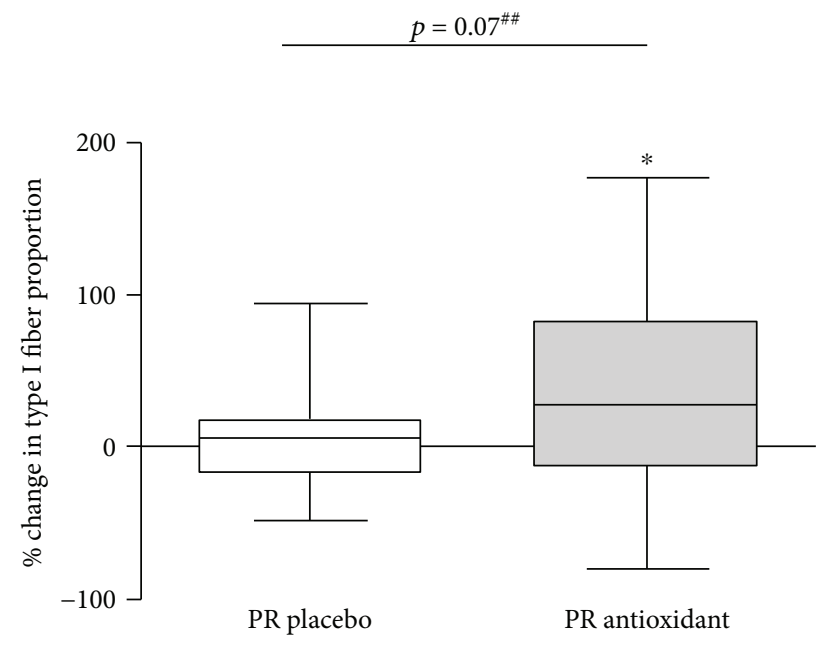

(b)

FIGURE 2: Box-and-whisker plots for relative change (\%) in functional parameters: (a) quadriceps maximal voluntary contraction (QMVC) and (b) quadriceps type I oxidative fiber proportion. ${ }^{*} p<0.05,{ }^{* *} p<0.01$, and ${ }^{* * *} p<0.001$. ${ }^{*}$ Group effect adjusted on the corticosteroid treatment. ${ }^{\# \#}$ Group effect adjusted on the baseline value and interaction term.

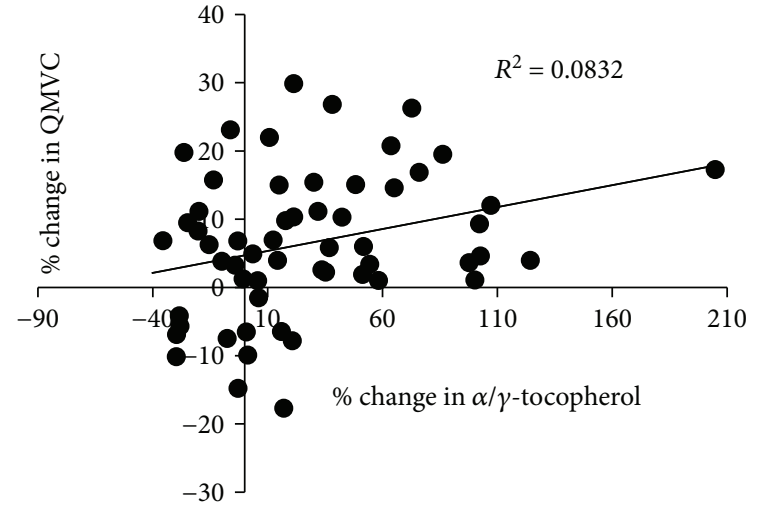

(a)

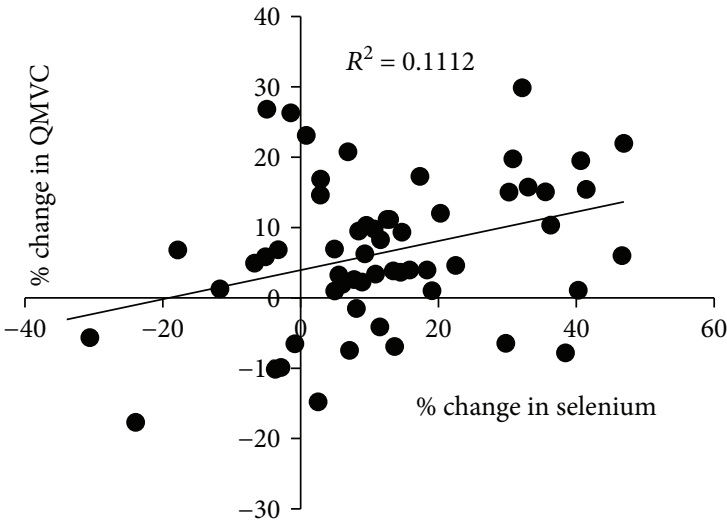

(b)

FIgURE 3: Linear regression analysis. The change (in \%) in quadriceps maximal voluntary contraction (QMVC) was independently correlated with the change in the $\alpha$-tocopherol $/ \gamma$-tocopherol ratio $(r=0.39, p<0.05)$ in all COPD patients $(\mathrm{a})$, and the change (in \%) in quadriceps maximal voluntary contraction (QMVC) was independently correlated with the change in selenium $(r=-0.64, p<0.001)$ in all COPD patients (b).

selenium deficit and the increases in the $\alpha$-tocopherol $/ \gamma$ tocopherol ratio and selenium in the supplemented COPD patients, the oxidative fiber increase in this group could be imputed to supplementation.

\section{Conclusions}

In this double-blind randomized placebo-controlled trial, antioxidant supplementation associating vitamin $\mathrm{E}$, vitamin $\mathrm{C}$, zinc, and selenium was administered to COPD patients for 28 days in combination with pulmonary rehabilitation supplementation. This combination failed to improve the quadriceps endurance (primary outcome). Yet, it improved quadriceps muscle strength and serum total protein, and type I fiber proportion showed a trend toward increase. Thus, this is the first study to suggest that efficient antioxidant supplementation results in a clinically significant effect on a relevant outcome in PR (i.e., muscle strength), as well as other specific adaptations not explained by training alone. This study opens a field for more targeted antioxidant supplementation and "add-on" nutritional interventions in combination with exercise training during PR. Ancillary muscle analysis would provide greater understanding of the cell pathways involved in these skeletal muscle adaptations, such as a reduction in inflammation and/or oxidative stress or an adaptation of the muscle oxidative phenotype.

\section{Data Availability}

The data used to support the findings of this study are available from the corresponding author upon request. 


\section{Disclosure}

The manuscript is based on Dr. Jonathan Maury's thesis. This work was presented at the London ERS Congress 2016 as a "late-breaking abstract": "Antioxidant Supplementation in Chronic Obstructive Pulmonary Disease (COPD): A Relevant Way to Optimize Pulmonary Rehabilitation Effects." Maury J, Gouzi F, Héraud N, et al. European Respiratory Journal 2016 48: PA714; doi:10.1183/13993003.congress2016.PA714.

\section{Conflicts of Interest}

The other authors declare that they have no conflict of interest.

\section{Authors' Contributions}

Fares Gouzi and Jonathan Maury contributed equally to this work.

\section{Acknowledgments}

The authors gratefully acknowledge M. Thibault Sutra for laboratory help during the study, Azziza Seddiki for rigorous monitoring of the study, and C. Stott for critical reading of the manuscript. We would also like to acknowledge the team of La Solane Pulmonary Rehabilitation Center for their contribution to this work, particularly Jacques Desplan, $\mathrm{MD}$, who made this study possible. F.G. received grants from the following companies: APARD, SOS Oxygene, Bastide Medical, and Agir à Dom/Homair. J.Ma. was supported by a CIFRE grant (Conventions Industrielles de Formation par la Recherche) from 5 Santé/Fontalvie Corporation and the French Association Nationale de la Recherche et de la Technologie. This study was supported by joint grants from the French Ministry of Public Health (Programme Hospitalier de Recherche Clinique Interrégional PHRCI, 2011), Montpellier University Hospital, and 5 Santé/Fontalvie Corporation, Toulouges, France.

\section{References}

[1] C. F. Vogelmeier, G. J. Criner, F. J. Martinez et al., "Global strategy for the diagnosis, management, and prevention of chronic obstructive lung disease 2017 report. GOLD executive summary," American Journal of Respiratory and Critical Care Medicine, vol. 195, no. 5, pp. 557-582, 2017.

[2] F. Maltais, M. Decramer, R. Casaburi et al., "An official American Thoracic Society/European Respiratory Society statement: update on limb muscle dysfunction in chronic obstructive pulmonary disease," American Journal of Respiratory and Critical Care Medicine, vol. 189, no. 9, pp. e15-e62, 2014.

[3] M. A. Spruit, S. J. Singh, C. Garvey et al., "An official American Thoracic Society/European Respiratory Society statement: key concepts and advances in pulmonary rehabilitation," American Journal of Respiratory and Critical Care Medicine, vol. 188, no. 8, pp. e13-e64, 2013.

[4] F. Gouzi, C. Prefaut, A. Abdellaoui et al., "Blunted muscle angiogenic training-response in COPD patients versus sedentary controls," The European Respiratory Journal, vol. 41, no. 4, pp. 806-814, 2013.

[5] J. De Brandt, M. A. Spruit, W. Derave, D. Hansen, L. E. Vanfleteren, and C. Burtin, "Changes in structural and metabolic muscle characteristics following exercise-based interventions in patients with COPD: a systematic review," Expert Review of Respiratory Medicine, vol. 10, no. 5, pp. 521-545, 2016.

[6] P. Pomies, M. Blaquiere, J. Maury, J. Mercier, F. Gouzi, and M. Hayot, "Involvement of the FoxO1/MuRF1/atrogin-1 signaling pathway in the oxidative stress-induced atrophy of cultured chronic obstructive pulmonary disease myotubes," PLoS One, vol. 11, no. 8, article e0160092, 2016.

[7] A. Couillard and C. Prefaut, "From muscle disuse to myopathy in COPD: potential contribution of oxidative stress," The European Respiratory Journal, vol. 26, no. 4, pp. 703-719, 2005.

[8] C. Koechlin, A. Couillard, D. Simar et al., "Does oxidative stress alter quadriceps endurance in chronic obstructive pulmonary disease?," American Journal of Respiratory and Critical Care Medicine, vol. 169, no. 9, pp. 1022-1027, 2004.

[9] H. Blaser, C. Dostert, T. W. Mak, and D. Brenner, "TNF and ROS crosstalk in inflammation," Trends in Cell Biology, vol. 26, no. 4, pp. 249-261, 2016.

[10] M. Kozakowska, K. Pietraszek-Gremplewicz, A. Jozkowicz, and J. Dulak, "The role of oxidative stress in skeletal muscle injury and regeneration: focus on antioxidant enzymes," Journal of Muscle Research and Cell Motility, vol. 36, no. 6, pp. 377-393, 2015.

[11] A. Azzi, "Many tocopherols, one vitamin E," Molecular Aspects of Medicine, vol. 61, pp. 92-103, 2018.

[12] Z. Huang, A. H. Rose, and P. R. Hoffmann, "The role of selenium in inflammation and immunity: from molecular mechanisms to therapeutic opportunities," Antioxidants \& Redox Signaling, vol. 16, no. 7, pp. 705-743, 2012.

[13] M. van Dijk, F. J. Dijk, A. Hartog et al., "Reduced dietary intake of micronutrients with antioxidant properties negatively impacts muscle health in aged mice," Journal of Cachexia, Sarcopenia and Muscle, vol. 9, no. 1, pp. 146-159, 2018.

[14] C. van Dronkelaar, A. van Velzen, M. Abdelrazek, A. van der Steen, P. J. M. Weijs, and M. Tieland, "Minerals and sarcopenia; the role of calcium, iron, magnesium, phosphorus, potassium, selenium, sodium, and zinc on muscle mass, muscle strength, and physical performance in older adults: a systematic review," Journal of the American Medical Directors Association, vol. 19, no. 1, pp. 6-11.e3, 2018.

[15] E. Mocchegiani, L. Costarelli, R. Giacconi et al., "Vitamin E-gene interactions in aging and inflammatory age-related diseases: implications for treatment. A systematic review," Ageing Research Reviews, vol. 14, pp. 81-101, 2014.

[16] E. Mocchegiani, L. Costarelli, R. Giacconi et al., "Micronutrient-gene interactions related to inflammatory/immune response and antioxidant activity in ageing and inflammation. A systematic review," Mechanisms of Ageing and Development, vol. 136-137, pp. 29-49, 2014.

[17] S. Saboori, S. Shab-Bidar, J. R. Speakman, E. Yousefi Rad, and K. Djafarian, "Effect of vitamin E supplementation on serum C-reactive protein level: a meta-analysis of randomized controlled trials," European Journal of Clinical Nutrition, vol. 69, no. 8, pp. 867-873, 2015. 
[18] A. Pingitore, G. P. P. Lima, F. Mastorci, A. Quinones, G. Iervasi, and C. Vassalle, "Exercise and oxidative stress: potential effects of antioxidant dietary strategies in sports," Nutrition, vol. 31, no. 7-8, pp. 916-922, 2015.

[19] V. Paschalis, A. A. Theodorou, A. Kyparos et al., "Low vitamin $\mathrm{C}$ values are linked with decreased physical performance and increased oxidative stress: reversal by vitamin $C$ supplementation," European Journal of Nutrition, vol. 55, no. 1, pp. 45-53, 2016.

[20] J. Maury, F. Gouzi, P. De Rigal et al., "Heterogeneity of systemic oxidative stress profiles in COPD: a potential role of gender," Oxidative Medicine and Cellular Longevity, vol. 2015, Article ID 201843, 11 pages, 2015.

[21] L. Laviolette, L. C. Lands, N. Dauletbaev et al., "Combined effect of dietary supplementation with pressurized whey and exercise training in chronic obstructive pulmonary disease: a randomized, controlled, double-blind pilot study," Journal of Medicinal Food, vol. 13, no. 3, pp. 589-598, 2010.

[22] G. Biró, K. F. A. M. Hulshof, L. Ovesen, J. A. A. Cruz, and for the EFCOSUM Group, "Selection of methodology to assess food intake," European Journal of Clinical Nutrition, vol. 56, no. S2, pp. S25-S32, 2002.

[23] J. M. Seymour, M. A. Spruit, N. S. Hopkinson et al., "The prevalence of quadriceps weakness in COPD and the relationship with disease severity," The European Respiratory Journal, vol. 36, no. 1, pp. 81-88, 2010.

[24] J. Fontaine, J. Neve, A. Peretz, P. Capel, and J. P. Famaey, "Effects of acute and chronic prednisolone treatment on serum zinc levels in rats with adjuvant arthritis," Agents and Actions, vol. 33, no. 3-4, pp. 247-253, 1991.

[25] M. A. Minetto, A. Botter, F. Lanfranco, M. Baldi, E. Ghigo, and E. Arvat, "Muscle fiber conduction slowing and decreased levels of circulating muscle proteins after short-term dexamethasone administration in healthy subjects," The Journal of Clinical Endocrinology \& Metabolism, vol. 95, no. 4, pp. 1663-1671, 2010.

[26] E. Passerieux, M. Hayot, A. Jaussent et al., "Effects of vitamin $\mathrm{C}$, vitamin E, zinc gluconate, and selenomethionine supplementation on muscle function and oxidative stress biomarkers in patients with facioscapulohumeral dystrophy: a doubleblind randomized controlled clinical trial," Free Radical Biology \& Medicine, vol. 81, pp. 158-169, 2015.

[27] L. Puente-Maestu, P. Palange, R. Casaburi et al., "Use of exercise testing in the evaluation of interventional efficacy: an official ERS statement," The European Respiratory Journal, vol. 47, no. 2, pp. 429-460, 2016.

[28] D. Shrikrishna, R. J. Tanner, J. Y. Lee et al., “A randomized controlled trial of angiotensin-converting enzyme inhibition for skeletal muscle dysfunction in COPD," Chest, vol. 146, no. 4, pp. 932-940, 2014.

[29] C. A. Camillo, C. R. Osadnik, H. van Remoortel, C. Burtin, W. Janssens, and T. Troosters, "Effect of "add-on" interventions on exercise training in individuals with COPD: a systematic review," ERJ Open Research, vol. 2, no. 1, 2016.

[30] W. H. Liao, J. W. Chen, X. Chen et al., "Impact of resistance training in subjects with COPD: a systematic review and meta-analysis," Respiratory Care, vol. 60, no. 8, pp. 11301145, 2015.

[31] L. Pan, Y. Guo, X. Liu, and J. Yan, "Lack of efficacy of neuromuscular electrical stimulation of the lower limbs in chronic obstructive pulmonary disease patients: a meta-analysis," Respirology, vol. 19, no. 1, pp. 22-29, 2014.
[32] N. V. Margaritelis, V. Paschalis, A. A. Theodorou, A. Kyparos, and M. G. Nikolaidis, "Antioxidants in personalized nutrition and exercise," Advances in Nutrition, vol. 9, no. 6, pp. 813-823, 2018.

[33] C. van de Bool, E. P. A. Rutten, A. van Helvoort, F. M. E. Franssen, E. F. M. Wouters, and A. M. W. J. Schols, "A randomized clinical trial investigating the efficacy of targeted nutrition as adjunct to exercise training in COPD," Journal of Cachexia, Sarcopenia and Muscle, vol. 8, no. 5, pp. 748758, 2017.

[34] J. M. Hofford, L. Milakofsky, W. H. Vogel, R. S. Sacher, G. J. Savage, and S. Pell, "The nutritional status in advanced emphysema associated with chronic bronchitis: a study of amino acid and catecholamine levels," American Review of Respiratory Disease, vol. 141, 4, Part 1, pp. 902-908, 1990.

[35] C. E. Bolton, A. A. Ionescu, K. M. Shiels et al., "Associated loss of fat-free mass and bone mineral density in chronic obstructive pulmonary disease," American Journal of Respiratory and Critical Care Medicine, vol. 170, no. 12, pp. 1286-1293, 2004.

[36] J. M. Zingg, "Vitamin E: a role in signal transduction," Annual Review of Nutrition, vol. 35, no. 1, pp. 135-173, 2015.

[37] M. Rederstorff, A. Krol, and A. Lescure, "Understanding the importance of selenium and selenoproteins in muscle function," Cellular and Molecular Life Sciences, vol. 63, no. 1, pp. 52-59, 2006.

[38] K. E. Hill, A. K. Motley, X. Li, J. M. May, and R. F. Burk, "Combined selenium and vitamin E deficiency causes fatal myopathy in guinea pigs," The Journal of Nutrition, vol. 131, no. 6, pp. 1798-1802, 2001.

[39] H. Ishihara, F. Kanda, T. Matsushita, K. Chihara, and K. Itoh, "White muscle disease in humans: myopathy caused by selenium deficiency in anorexia nervosa under long term total parenteral nutrition," Journal of Neurology, Neurosurgery \& Psychiatry, vol. 67, no. 6, pp. 829-830, 1999.

[40] T. Rannem, K. Ladefoged, E. Hylander et al., "The effect of selenium supplementation on skeletal and cardiac muscle in selenium-depleted patients," Journal of Parenteral and Enteral Nutrition, vol. 19, no. 5, pp. 351-355, 1995.

[41] A. J. Zamora, F. Tessier, P. Marconnet, I. Margaritis, and J. F. Marini, "Mitochondria changes in human muscle after prolonged exercise, endurance training and selenium supplementation," European Journal of Applied Physiology and Occupational Physiology, vol. 71, no. 6, pp. 505-511, 1995. 


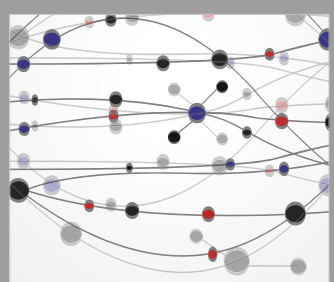

The Scientific World Journal
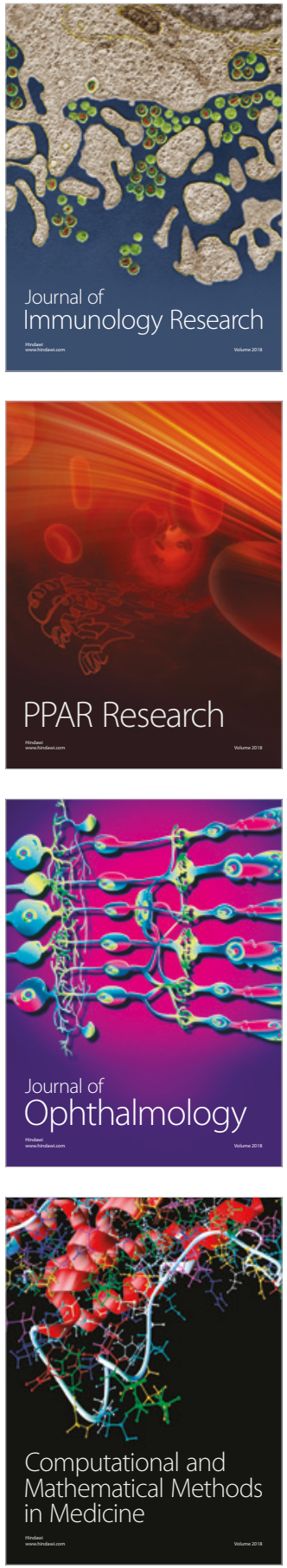

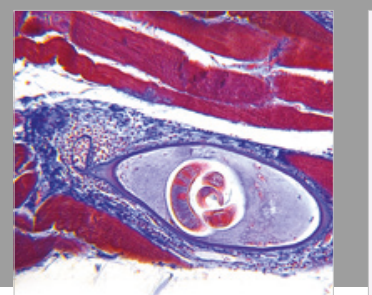

Gastroenterology Research and Practice

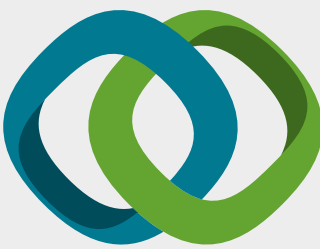

\section{Hindawi}

Submit your manuscripts at

www.hindawi.com
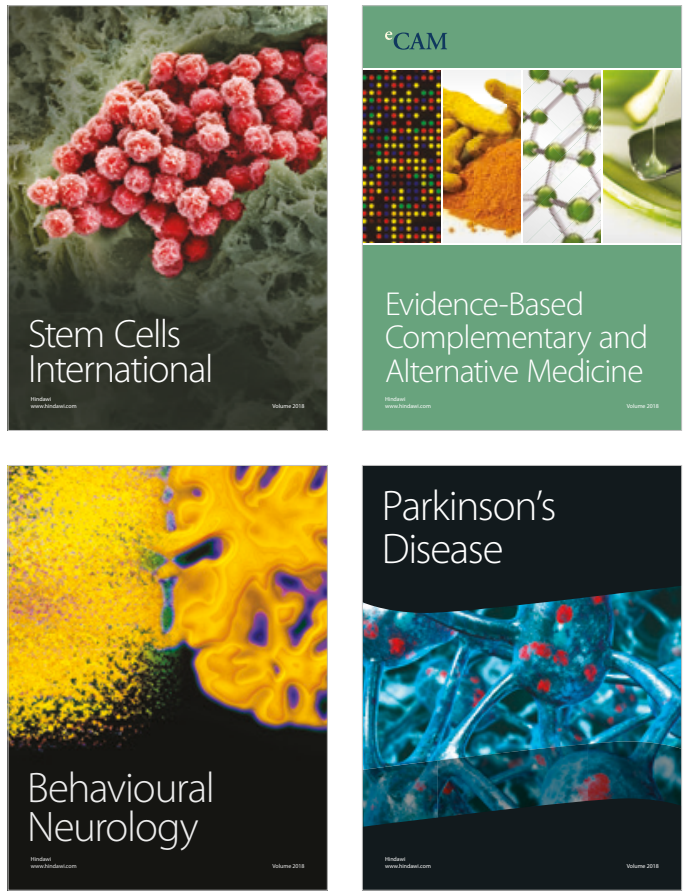

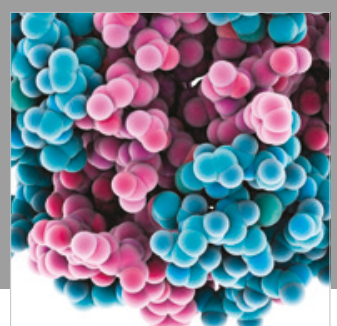

ournal of

Diabetes Research

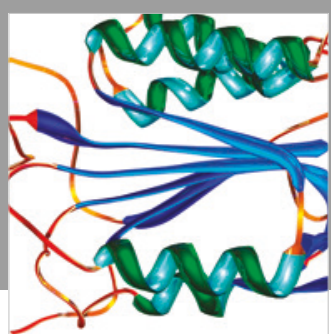

Disease Markers
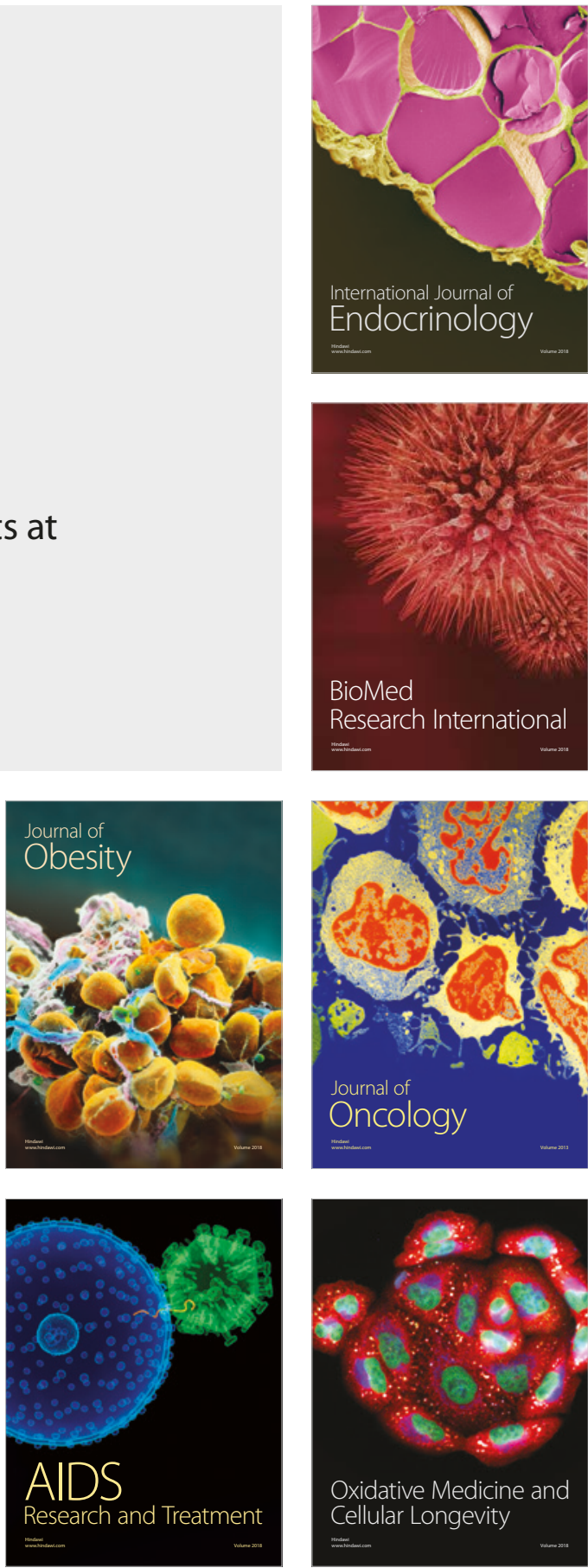